\title{
Effectiveness of an Interventional Educational Tool on Certain Overweight and Obesity Parameters Among the General Public in Sungai Petani, Kedah Darul Aman, Malaysia
}

\author{
Sam Aaseer Thamby ${ }^{1,2}$, Azmi Bin Sarriff², Ng Yen Ping ${ }^{1}$, Dayana Nicholas ${ }^{1}$, Balasubramanian Ganesh Pandian ${ }^{1}$, Sireesha Paruchuri $^{3}$ \\ ${ }^{1}$ Faculty of Pharmacy, AIMST University, MALAYSIA. \\ ${ }^{2}$ Faculty of Pharmacy, Universiti Sains Malaysia, MALAYSIA. \\ ${ }^{3}$ Department of Pharmacy Practice, Vaagdevi College of Pharmacy Warangal, Telangana, INDIA.
}

\begin{abstract}
Background: Overweight and obesity prevalence are increasing in Malaysia. This epidemic has affected a very high number of children and young adults. This poses a serious health hazard, not only presently, but also for the future Malaysian adult population. Interventions aimed at educating and informing the public about the hazards of being overweight and/or obese is one of the best shots at combating this issue. Methods: This exploratory, prospective study employed a customized questionnaire (study instrument) and pre-validated handout (interventional tool). The main objective of this study was to evaluate the effectiveness of the interventional tool in improving the knowledge, dietary habits and physical activities of the study sample. A change in the scores pertaining to knowledge, dietary habits and physical activities, would be indicative of the interventional tool's effectiveness. Results: 112 males and 439 females participated in this study. The point prevalence of overweight and obesity combined was $70.07 \%$. The respondents' weight, BMI, knowledge, dietary habits and physical activities did improve significantly during the course of the study. The percent-
\end{abstract}

age values revealed an increase in weight, $\mathrm{BMI}$ and all the three domains assessed. On comparing P3 v P1, the percentage value increase (improvement) in weight, BMI, knowledge, dietary habits, physical activities, and total score was 10, 9, 35, 43.3, 17.85 and 35.7 respectively. Conclusion: The score grades of the respondents for the same parameters also showed an increase in 'moderate' and 'good' grades in P2, an increase in 'good' grades in P3, while the number of 'poor' grades which were high during the baseline study dropped markedly at the conclusion of P3.

Key words: Overweight, Obesity, Body Mass Index, Interventional tool, Spacedrepetition.

Correspondence:

Mr. Sam Aaseer Thamby, Faculty of Pharmacy; AIMST University, MALAYSIA.

Phone: 006-0164266240

Email: sam_thamby@aimst.edu.my

DOI: 10.5530/jyp.2017.9.112

\section{INTRODUCTION}

Obesity, is defined as 'abnormal or excessive fat accumulation that may impair health'and is a highly prevalent and serious public problem. The prevalence of obesity amoung American adult is $35.7 \%$ and increases rates of obesity-related illnesses including cardiovascular disease (CVD). The BMI cutoffs in a multiethnic Malaysian population that included people of Malay, Chinese, and Indian origins were 23.0 and $24.0 \mathrm{~kg} / \mathrm{m}^{2}$ respectively. ${ }^{1}$ Fournier et al., observed that, $22 \%$ of the representative study Malaysian population were overweight and which may increase the incidence of obesity and increase the prevalence of cardiovascular illnesses. ${ }^{2}$ Excess body fat, especially abdominal fat, increases the risk of many conditions, including insulin resistance and Type-II diabetes. ${ }^{3}$ The relationship between physical activity and obesity is a convolutedcase. It is a general notion that low physical activity levels are associated with body weight gain over time. ${ }^{4}$

The National Health and Morbidity Survey (NHMS, Malaysia) 1996 reported $15.1 \%$ of adult males were overweight and $2.9 \%$ obese, while $17.9 \%$ and $5.7 \%$ adult females were overweight and obese respectively. The report stated that there existed very negligible differences between rural and urban populations, the Malays and Indians had higher incidences as compared to Chinese. ${ }^{5}$ The financial implications of overweight and obesity epidemic in Malaysia will inflict a huge burden on the human and economic resources and are liable to disturb priorities in the health care or other sectors (Ismail et al., 2002). The NHMS Report 2015 revealed $33.4 \%$ and $30.6 \%$ of a representative Malaysian young adults' population were overweight and obese respectively. $25.3 \%$ of the population had BMI in the range $27.5-34.9 \mathrm{~kg} / \mathrm{m}^{2}$. The NHMS Report 2015 showed that the national prevalence of overweight, obesity and abdominal obesity had increased by $0.6 \%, 2.6 \%$ and $2.0 \%$ respectively, as compared to the previous findings of NHMS $2011 .^{6}$

Malaysia has been witnessing an astronomical increase in the proportion of overweight and obese patients. Despite numerous efforts undertaken by the various healthcare organizations and the Ministry of Health, the percentages of overweight and obese cases still keep on increasing. The National Health and Morbidity Survey 2015 stated that 33.4\% of the Malaysian population was overweight, while the national prevalence of obesity was $30.6 \%$ (NHMS, 2015). A disturbing fact is that when compared to the previous NHMS report (i.e., in 2011), a $0.6 \%$ increase in overweight and $2.6 \%$ increase in obesity cases among Malaysians was noted. Health campaigns, patient counselling and various strategies have not been as successful as envisaged by the healthcare authorities and the Ministry of Health. ${ }^{6}$ Hence, the present study was planned to assess (i) the point prevalence of overweight and obesity among the selected representative sample;(ii) the changes in knowledge, dietary habits, and physical activities among the sample; and (iii) to evaluate the effectiveness of the interventional tool in improving theknowledge, dietary habits and physical activities of the study sample with regardto overweight and obesity.

This is an open access article distributed under the terms of the Creative Commons Attribution-NonCommercial-ShareAlike 4.0 License, which allows others to remix, tweak, and build upon the work non-commercially, as long as the author is credited and the new creations are licensed under the identical terms. 


\section{MATERIAL AND METHODS}

This study design is a exploratory, prospective, descriptive, longitudinal cohortstudy comprising three main phases, with the variable(s) knowledge, dietary habits, physical activity practice among the study population being recorded at each point of time, in lieu of overweight and obesity. This was done in order to obtain the point prevalence of overeweight and obesity among the sample. The study population were sourced from among Sungai Petani residents (Kedah state, Malaysia) over the study period. The gap between each phase was 6 months. This was based on the premise that a time period of 4-6 months was necessary to note any defined change in BMI.Figure 1 depicts the study flow at the three different phases.

Malaysian citizens residing in Sungai petani for the last three generations, aged between $18-30$ years with BMI of $>25.0$ were included in the study. Expatriates, subject with age of $>18$ and $<30$ with BMI $>24.99$, pregenet mother, nursing women and persion with know STD were excluded from the study.

Convenience sampling technique was employed to source the 551 respondents. Prior to commencing the baseline study and distributing the questionnaires, the informed consent for participating in the study was obtained from all the participants. The self-administered questionnaire (in two versions, either English or Bahasa Melayu) was distributed to the subjects, based on their preference and degree of comfort with the language. Once the required number of targets was sourced, the researcher (along with the trained data collectors and/or interviewers) approached them in person during each phase, to distribute the questionnaire, collect back the completed questionnaires and distribute the handout. Contact with each respondent was initiated during the last week of month five between each phase. The researcher along with the trained data collectors met the respondents in person, assessed their weight, and calculated their BMIs. The study tool was then distributed to each respondent to obtain their responses. This was followed by distribution of the customized handout to each respondent for aiding memory retention. The same strategy was utilized for two more phases (Phases-II and III), spaced 6 months apart. The data from each phase was assessed to facilitate comparative evaluation. The logistical support for the study was provided by the trained data collectors and interviewers, who were actively involved in each of the three phases with respect to the distribution of the questionnaires, retrieval of the completed questionnaires, and distribution of the handouts to the respondents.

The scoring grades attributed to each domain and overall, were adopted from earlier KAP studies. Based on the original Bloom's cut off points, assessmentwith a score of $80-100 \%$ correct response meant a good KAP score, a score of $60-79 \%$ put the scorer in a level ofsatisfactory, average or moderateKAP score and a score lessthan $60 \%$ of correct response as poor. This was utilized for the scores pertaining to each domain of Knowledge, Attitude and Perception or Practice andoverall score wherever necessary. ${ }^{7-9}$

The data of dependent variables were not normally distributed, as revealed by the Shapiro-Wilk test. Though the data was numerical, the non-parametric tests wereemployed for data analyses. As the data were non-parametric, the median was used to describe the weight, height variables. Frequency was used to describe the gender and ethnicity data. The BMI frequencies were also expressed for the three phases. The Friedman ANOVA test was used to compare the BMI and weight differences through the three phases. The differences of the variables between each phase (paired; P1 vs P2; P1 vs P3; P2 vs P3) was analysed by conducting Post-hoc analysis-Wilcoxon Signed Rank test. The Chi-Square test was used to compare gender, ethnicity and BMI.

\section{Statistical analysis}

Friedman ANOVA was used to look at the differences between several related groups. The same groups of patients were involved in all the three phases. If the Friedman ANOVA test result was significant, Post-hoc analysis by using Wilcoxon-signed rank test was conducted to determine where the differences lie. For questions with 'Yes' and 'No' options only, Chi-Square test was used to test the statistical significance of the said frequencies. The association between the independent variables (gender and ethnicity) and Section-A were assessed by Pearson's Chi-Square test. Median was used to compare the total scores of sections- $\mathrm{A}, \mathrm{B}$ and $\mathrm{C}$ in each of the three phases as the data was non-parametric. The statistical significance of the total scores pertaining to sections- $\mathrm{A}, \mathrm{B}$ and $\mathrm{C}$ in each of the three phases was assessed by Friedman ANOVA test. The Wilcoxon Signed Ranks test (Post-hoc analysis) was conducted to evaluate the statistical significance between each pair of the total score in the respective phases, i.e., paired; $\mathrm{P} 1$ vs $\mathrm{P} 2$; $\mathrm{P} 1$ vs $\mathrm{P} 3$; $\mathrm{P} 2$ vs $\mathrm{P} 3$ in sections- $\mathrm{A}$, $\mathrm{B}$ and $\mathrm{C}$ respectively.

The median was used to describe the total scores obtained in each of the three phases. The statistical significance between the total scores in each of the three phases was assessed by Friedman ANOVA test. The Wilcoxon Signed Ranks test (Post-hoc analysis) was conducted to evaluate the statistical significance between each pair of the total score in the respective phases (paired; $\mathrm{P} 1$ vs $\mathrm{P} 2$; $\mathrm{P} 1$ vs $\mathrm{P} 3$; $\mathrm{P} 2$ vs $\mathrm{P} 3$ in sections- $\mathrm{A}, \mathrm{B}$ and $\mathrm{C}$ respectively).

\section{RESULTS}

The median age (in years), median height (in cms) and weight (in kgs) of the respondents were 20 years, $161 \mathrm{cms}$ and $70 \mathrm{kgs}$ respectively. Table 1 shows the median scores, IQR and associated values observed in sections $\mathrm{A}, \mathrm{B}$ and $\mathrm{C}$ during the three phases of the study. The Friedman ANOVA test was followed by the Wilcoxon Signed Ranks test. The upward surge in these median scores was revealed to bestatistically significant, $\chi^{2}(2)$ $=1020.620, p<.001$ and $\chi^{2}(2)=586.896, p<.001$ for section $\mathrm{A}$ and $\mathrm{C}$ respectively. The rise in the median scores was revealed to be statistically significant, $\chi^{2}(2)=1088.026, p<.00$ for section $\mathrm{B}$.

The total score was calculated by summating the score range of the three sections (knowledge, dietary habits assessment and physical activities domain), leading to an overall score range of $0-205$. Figure 2 depicts the overall median scores and ranks for the three phases. The Friedman test, which evaluated differences in median among the three phases, was significant $\chi^{2}(2)=964.004, p<0.001$ and the Kindall's coefficient of concordance, $(W=.32)$ indicating fairly strong differencesamong the three phases of the study. An increase in the median overall (total) scores of the respondents from P1 to P3 can be observed. This indicates an improvement and a paradigm shift towards a better outcome with regard to their weight, BMI, knowledge, dietary habits and physical activities. A marked decrease in weight and BMI can be observed from P1 to P3. While, the knowledge, dietary habits and physical activities of the respondents improved conspicuously during the course of the study period.

The effect of the interventional tool in improving the knowledge, dietary habits, physical activities, and subsequently, the weight and BMI of the respondents was calculated. Table 2 shows the percentage value differences for each section (A, B and $\mathrm{C}$ ) and the total score, along with the weight and BMI variations.

\section{DISCUSSION}

Of the 551 respondents, 532 were overweight, 14 were obese (class-I), and 5 were obese (class-II). High prevalence of being overweight and obesity results from an intricate synergy between an inherited metabolic 
Table 1: Median scores of the respondents in each section of the study instrument, the affiliated score percentages and grade

\begin{tabular}{cccccc}
\hline SECTION & & P1 & P2 & P3 & p value \\
\hline 'A' & Median & 20.00 & 25.00 & 27.00 & $<0.001^{*, * *}$ \\
(Knowledge) & IQR & 3.00 & 2.00 & 0.00 & \\
& Score \% & $66.6 \%$ & $83.3 \%$ & $90.0 \%$ & \\
& Score grade & Moderate & Good & Good & \\
& Mean Rank & $1.01^{*}$ & $2.12^{*}$ & $2.87^{*}$ & \\
'B' & Median & 90.00 & 112.00 & 129.00 & $<0.001^{*, * *}$ \\
(Dietary habits & IQR & 22.5 & 21.0 & 17.0 & \\
assessment) & Score \% & $64.7 \%$ & $80.6 \%$ & $92.8 \%$ & \\
& Score grade & Moderate & Good & Good & \\
& Mean Rank & $1.01^{*}$ & $2.00^{*}$ & $3.00^{*}$ & \\
'C' & Median & 28.00 & 31.00 & 33.00 & $<0.001^{*, * *}$ \\
activities) & IQR & 8.0 & 5.0 & 4.0 & \\
& Score \% & $77.7 \%$ & $86.1 \%$ & $91.6 \%$ & \\
& Score grade & Moderate & Good & Good & \\
& Mean Rank & $1.20^{*}$ & $2.17^{*}$ & $2.63^{*}$ & \\
\hline
\end{tabular}

*Friedman ANOVA; **Wilcoxon Signed Ranks.

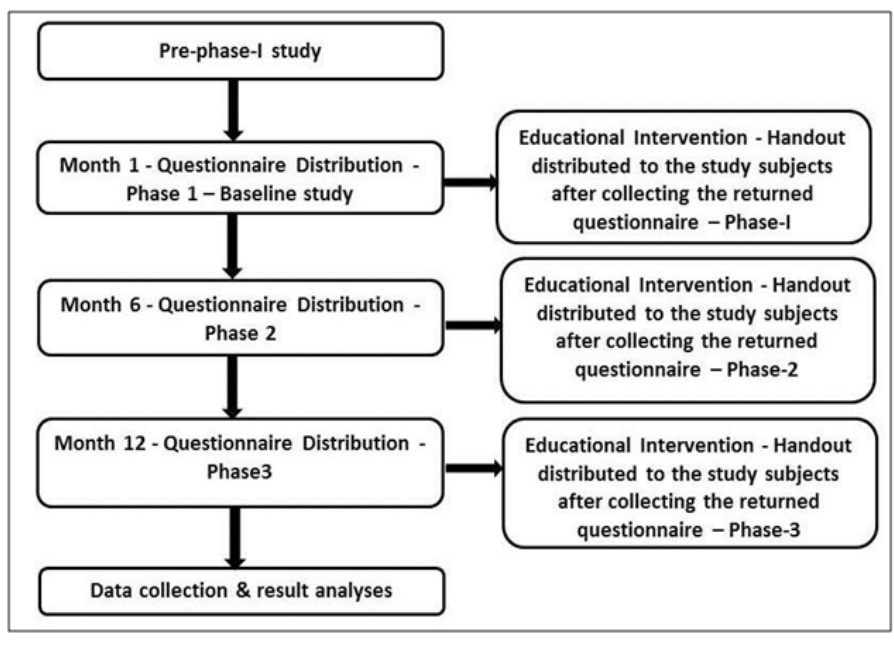

Figure 1: Study flow during the the three phases.

Table 2: Percentage value differences for each phase

\begin{tabular}{cccccccc}
\hline \multirow{2}{*}{ Domains } & \multicolumn{5}{c}{ Scores } & \multicolumn{5}{c}{ Percentage Values } & \multirow{2}{*}{ p value } \\
\cline { 2 - 6 } & P1 & P2 & P3 & P2vP1 & P3vP2 & P3vP1 & \\
\hline $\begin{array}{c}\text { Sec. A } \\
\text { (Knowledge) }\end{array}$ & 20 & 25 & 27 & 25 & 8 & 35 & $<0.001^{*, * *}$ \\
$\begin{array}{c}\text { Sec. B } \\
\text { (Dietary Habits) }\end{array}$ & 90 & 112 & 129 & 24.4 & 15.17 & 43.3 & $<0.001^{*, * *}$ \\
$\begin{array}{c}\text { Sec. C(Physical } \\
\text { Activities) }\end{array}$ & 28 & 31 & 33 & 10.7 & 6.45 & 17.85 & $<0.001^{*, * *}$ \\
Total score & 140 & 171 & 190 & 22.1 & 11.1 & 35.7 & $<0.001^{*, * *}$ \\
Weight & 70 & 67 & 63 & 4.28 & 5.97 & 10.0 & $<0.001^{*, * *}$ \\
BMI $^{*}$ & 26.64 & 25.56 & 24.24 & 4.05 & 5.16 & 9.0 & $<0.001^{*, * *}$ \\
\hline
\end{tabular}

The values shown for the weight and BMI percentage values are absolute. ${ }^{\star}$ Friedman ANOVA; ${ }^{* \star}$ Wilcoxon Signed Ranks.

proneness to being overweight, and changes in the individual's lifestyle which usually go hand in hand with economic growth and transition. ${ }^{10}$

Many studies conducted in Malaysia augment the findings observed in the current study. The National Health and Morbidity Survey (2015), conducted throughout Malaysia, assessing the status of numerous pathological states revealed that $33.4 \%$ of the population overweight, while the national prevalence of obesity stood at a staggering 30.6\%. ${ }^{6}$ The MANS (Malaysian Adults Nutrition Survey) study was conducted in the year 2009 on a very large scale, involving clusters of 3,441 women and 6,775 men aged 18 - 59 years. Their findings revealed that the overall mean body weight was $62.65 \mathrm{~kg}$ and BMI was $24.37 \mathrm{~kg} / \mathrm{m}^{2}$. It can be observed that during that year itself, the body weight and the corresponding BMI was in line with the preceding scenario for overweight and obesity. The maximum number of cases was observed in respondents who were in the fourth decade. Their findings revealed that $12.15 \%$ (1242) of the sample were obese, while $26.71 \%$ (2729) were overweight. Regarding the gender, females were more obese (14.66\%), while males were more overweight (28.55\%). Prevalence of obesity was highest among the Malays (15.28\%), while Indians had the highest overweight cases (31.01\%).
Those with only primary education had the highest overweight prevalence $(31.90 \%) .{ }^{11}$

In a study, the relationship between lifestyle factors (like eating patterns, dietary intake, and physical activity) and BMI were assessed by using a cross-sectional survey. The participants aged 18-99, were 928 males and 889 females, from Wyoming, Idaho, and Montana. This study revealed that age wasn't a significant predictor of overweight or obesity risk. The respondents, irrespective of the gender and age were found to be at significantly greater risk for overweight $\left(\mathrm{BMI}>25 \mathrm{~kg} / \mathrm{m}^{2}\right)$ and obesity $\left(\mathrm{BMI}>30 \mathrm{~kg} / \mathrm{m}^{2}\right)$ when consuming carbonated beverages $(\mathrm{p}=0.0006$; $\mathrm{p}=0.0143)$, watching television excessively $(\mathrm{p}=0.0050 ; \mathrm{p}=0.0017)$, and self-assessment of requirement for increase in physical activity $(\mathrm{p}=0.001$; $\mathrm{p}=0.0001$ ). Significant associations were noted between consuming supersized portions $(\mathrm{p}=0.0035)$ and obesity; and, eating while engaged in other activities like TV viewing $(\mathrm{p}=0.0003) .{ }^{12}$ Results in this study are consistent with other studies implicating consumption of energy-dense foods coupled with decreased physical activity.

The relationship between knowledge and obesity among 292 adolescents was assessed in one study. There were $26 \%$ obese cases among 


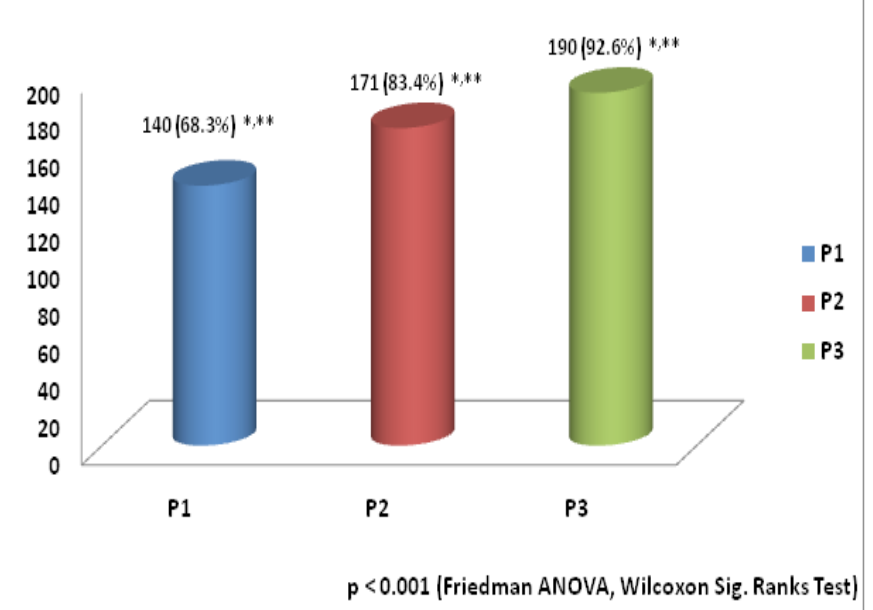

Figure 1: Study flow during the the three phases.

the respondents. This study revealed no significant differences between knowledge and improvement in status regarding overweight and obesity. Overall, the nutrition more or less was the same between the obese and non-obese respondents. ${ }^{13}$ This result was in contradiction to a study conducted by in Busan, Korea. The effects of gender, obesity rate, nutrition knowledge and dietary attitude on the dietary self-efficacy of adolescents were studied. Their study revealed that higher the nutrition-related knowledge and attitude towards food habits, higher was the dietary selfefficacy among the respondents. Knowledge regarding nutrition, food habits, overweight and obesity negated the indirect effects of obesity on dietary self-efficacy. ${ }^{14}$

Numerous factors immensely contribute to the overweight and obesity epidemic. The intake patterns, quantity and quality of foods consumed impact the energy equation, resulting in a 'positive energy balance' (wherein excess energy is stored in the body). A prospective study observed that increasing intake of fruits and vegetables was associated with a reduced risk of weight gain ( $\geq 25 \mathrm{kgs}$ ), or becoming obese ( $\geq 30 \mathrm{~kg}$ / $\left.\mathrm{m}^{2}\right) .{ }^{15} \mathrm{~A}$ similar result was observed in a study, wherein vegetables consumption on a daily basis was reported to reduce the risk of obesity. ${ }^{16}$

Overweight and obesity can result from a minor energy imbalance leading to a gradual, but persistent, weight gain over a prolonged time period. Physical activity plays a major part in energy imbalance, subsequently resulting in obesity. Decrease in physical activity levels compound or exacerbate over-nutrition effects on overweight and obese individuals. Physical activity is one of the vital components of energy balance. Promotion of a healthy lifestyle by incorporating more physical activities is mandatory for children and young adults as a lifelong positive healthy behaviour. $^{17}$

The impact of exercise and physical activities in management of overweight and obesity individuals were studied earlier. The study findings were that when $\mathrm{BMI} \geq 30$, the mortality risk due to Cardio Vascular Disorders (CVD) increased by $50-100 \%$. Weight loss in overweight and obese individuals decreased the risk factors for Diabetes Mellitus (DM) and CVD. Involvement in physical activities and exercises resulted in better adaptive responses by the body, causing better utilization of lipids. The impact of physical activities and exercises was that it counteracted the 'environment' to prevent these people from regaining weight. The researchers stated that regular low-endurance exercises were sufficient to achieve these outcomes. The use of physical activity to reduce weight is inversely related to patient's age and BMI, but directly related to the interventional education imparted. They concluded that direct, more intense face-to-face interaction between the HCP (pharmacist included) and the $\mathrm{o} / \mathrm{w}$ and/or obese patients at high risk of CVD. ${ }^{18}$
Physical activity leads to increased energy expenditure and fat loss, shields the body against the loss of lean body mass, improves efficiency of the cardiorespiratory system, minimizes cardiometabolic health risks which are obesity-related, and elicit perceptions of wellness. Improved oxygen transfer to muscle is a key feature of aerobic physical training, causing increased utilization of abundant fat stores instead of the limited glycogen stores. Moderately intense physical activities 30 minutes a day for 5 days every week is advocated. This activity if followed for month can contribute to $0.5 \mathrm{~kg}$ weight loss. Realistic attainable goals should be reinforced to the respondents, with regard to the anticipated exerciseinduced weight loss and the advantageous ramifications of exercise on cardiometabolic risks. Weight loss optimization involved increasing the exercise time span to $60 \mathrm{~min}$ for 5 days a week. Overweight and obesity can also result from a lack of daily habitual physical activity. Physical activities (walking, cycling, and stair climbing) should be urged. The level of education is positively associated with the engagement of physical activity in weight management, and inversely associated with the occurrence of serious comorbidities, age and degree of overweight. ${ }^{19}$

Sedentary lifestyles and decreased physical activities partially account for the association with the likelihood of being overweight or obese. Tremblay and co. concluded that there was definitive evidence from their study supporting the link between physical inactivity and obesity of Canadian children..$^{20}$ Sedentary lifestyles characterized by excessive TV viewing, computer games and snacking, as proved by numerous studies revealed their strong association with increased prevalence of obesity. ${ }^{20,21}$ On average, the respondents' increase / improvement in knowledge, dietary habits and physical activities was $35 \%, 43.3 \%$ and $17.85 \%$ respectively. The percentage values for each phase of each section were statistically significant $(\mathrm{p}<0.001$; Friedman ANOVA test and Wilcoxon Signed Ranks test). Among the three domains, it can be seen that the highest increase (in terms of the percentage values) was observed in section-'B' (dietary habits), followed by section-'A' (knowledge). However, the least increase in the percentage value was observed in section- ' $C$ ' (physical activities). It can be assumed / deduced that during the course of the study, the respondents were receptive to the interventional tool. This in effect, produced a change in their dietary habits and physical activities, resulting in improved scores during the phases.

An increase in the median overall (total) scores of the respondents from P1 to P3 can be observed. This indicates an improvement and a paradigm shift towards a better outcome with regard to their knowledge, dietary habits, physical activities, weight, and BMI. A marked decrease in weight and BMI can be observed from P1 to P3, while the knowledge, dietary habits and physical activities of the respondents improved conspicuously during the course of the study period. This can be attributed to three factors: (a) the study instrument and interventional tool, (b) respondents' motivation to continue through the study, and (c) the phenomenon of 'spaced repetition', which will be discussed at length towards the end of this section.

It must be noted that for effective weight management, improvements in the trifecta of knowledge, dietary habits, and physical activities are mandatory. The confluence of these three domains is a prominent feature in weight management. A group of researchers studied the improvement in nutrition-related knowledge and behaviour of urban Asian Indian school children, which was a core component of the Medical education for children/Adolescents for Realistic prevention of obesity and diabetes, and for healthy aGeing (MARG) study. 3128 children, 2241 parents and 831 teachers were the participants. The baseline knowledge was low in $75-94 \%$ of the groups. However, post-intervention, knowledge base increased in all groups $(\mathrm{p}<0.001)$. Their conclusions highlighted that major gaps exist in knowledge regarding core issues of obesity and overweight. They recommended this mode of educational intervention in future school-based curriculum and public health campaigns, in order 
Thamby et al.: Interventional educational tool's effectiveness on overweight and obesity parameters

to educate the individuals and improve their dietary habits and physical activities, in relation to overweight and obesity. ${ }^{22}$

This study has provided certain useful insights and future direction for the researchers, general public, and policy makers in combating overweight and obesity in Malaysia. The domains of knowledge, dietary habits and physical activities are pertinent to combating the overweight and obesity epidemic, as proven by this study. Future similar studies and health campaigns can opt for targeting the same domains. Increased involvement of families and education can be a focal point of future research works and health campaigns. A longer follow-up period with more phases would be beneficial for future similar studies. Interventional tools (handouts) are vital in these studies. However, distributing the handouts on the principles of spaced repetition can be more effective in increasing the respondents' healthy behaviours with regard to overweight and obesity. The current scientific knowledge can be expanded by all of these domains about the interrelated mechanisms and environmental factors causing health risks associated with overweight and obesity. Priority must be placed on preventing or atleast reducing the incidence rates, prevalence rates (point and/or period) of overweight and obesity among the younger generation. Educating school and university students regarding their dietary and physical activity environments can furnish a wave of advocates for healthy community environments. It will also be beneficial that future studies test the impact of weight and BMI reduction by different dietary modifications and increased levels of varied physical activities in reducing and/or preventing overweight and obesity-associated health risks across the Malaysian population.

\section{Limitations of the study}

The sampling technique utilized in this study was 'convenience sampling'. The study subjects cannot be controlled outside the boundaries of the study. The status of the respondents could only be assessed at the point of interaction during each phase of the study. The logistics of acquiring the data during subsequent phases was cumbersome and time-consuming.

\section{CONCLUSION}

This research employed qualitative methods to explore the respondents' status with regard to knowledge, dietary habits and physical activities in context of overweight and obesity. The pre-validated questionnaire and interventional tool were used to assess the respondents' in the three afore-mentioned domains, and to reinforce key concepts hoping to ensure positive, healthy trends in those domains respectively. A positive shift in the respondents' knowledge, dietary habits and physical activities were observed during the study. This was evident in the results pertaining to the objectives. The efficacy of the interventional tool could be indirectly determined by the positive variations in knowledge, dietary habits and physical activities of the respondents.

\section{ACKNOWLEDGEMENT}

The author acknowledges the contribution of the co-researchers, and Universiti Sains Malaysia to complete this research.

\section{CONFLICT OF INTEREST}

The authors have declared no conflict of interest.

\section{DISCLOSURE}

Part of the work was presented in 'PharmCare Symposium: Future Paradigm of Pharmaceutical Care Research' 5-6 th $^{\text {A }}$ August 2017 in Universiti Sains Malaysia, Penang.

\section{ABBREVIATION USED}

BMI: Body Mass Index; CVD: Cardio Vascular Diseases; HCP: Health Care Personnel; NHMS: National Health and Morbidity Survey; o/w: Overweight.

\section{REFERENCES}

1. Rao G, Powell-Wiley TM, Ancheta I, Hairston K, Kirley K, Lear SA, et al. Identification of obesity and cardiovascular risk in ethnically and racially diverse populations: A scientific statement from the American heart association. Circulation. 2015;132(5):457-72

2. Fournier T, Tibère L, Laporte $C$, Mognard E, Ismail MN, Sharif SP, et al. Eating patterns and prevalence of obesity. Lessons learned from the MalaysianFood Barometer. Appetite. 2016;107:362-71.

3. Clinical Guidelines on the Identification, Evaluation, and Treatment of Overweight and Obesity in Adults-The Evidence Report. National Institutes of Health. Obes Res. 1998;6(2):51S-209S. Review. Erratum in: Obes Res. 1998;6(6):464.

4. Hill JO, Melanson EL. Overview of the determinants of overweight and obesity: current evidence and research issues. Med Sci Sports Exerc. 1999;31(11 Suppl):S515-21.

5. Lim TO, Ding LM, Zaki M, Suleiman AB, Fatimah S, Siti S, et al. Distribution of body weight, height and body mass index in a national sample of Malaysian adults. Med J Malaysia. 2000;55(1):108-28.

6. National Health and Morbidity Survey (2015). Non-communicable diseases, risk factors and other health problems. Vol.2: MOH/S/IKU/52.15 (RR). Institute for Public Health. Ministry of Health, Malaysia. Retrieved from http://www.iku.gov. my/images/IKU/Document/REPORT/nhmsreport2015vol2.pdf. On 7 May 2014.

7. Counselling and communicating with men -Trainer's Manual; 2003 Engender Health. Retrieved from https://www.engenderhealth.org/files/pubs/gender/ mrhc-2/trainer/mrh_2t.pdf. on 19 May 2015.

8. Kaliyaperumal K. Guidelines for Conducting a Knowledge, Attitude and Practice (KAP) Study. Community Ophthalmol. 2004;4(1): 7-9.

9. Nahida A. Knowledge, attitude and practice on dengue fever. Retrieved from http://cphs.healthrepository.org/bitstream/123456789/1296/3/Thesis2007_Nahida.pdf. Accessed on 28/05/2014

10. Ismail MN. Obesity in Malaysia: prevalence and metabolic studies. In: Shetty PS, Gopalan C (eds). Diet, Nutrition and Chronic Disease: An Asian Perspective. London: Smith-Gordon \& Co. Ltd, 1998.

11. Azmi MY Jr, Junidah R, Mariam SA, Safiah MY, Fatimah S, Norimah AK, et al Body Mass Index (BMI) of Adults: Findings of the Malaysian Adult Nutrition Survey (MANS). Malays J Nutr. 2009;15(2):97-119.

12. Liebman M, Pelican S, Moore SA, Holmes B, Wardlaw MK, Melcher LM, et al Dietary intake, eating behavior, and physical activity-related determinants of high body mass index in rural communities in Wyoming, Montana, and Idaho. Int J Obes Relat Metab Disord. 2003;27(6):684-92.

13. Thakur N, D'Amico F. Relationship of nutrition knowledge and obesity in adolescence. Fam Med. 1999;31(2):122-7.

14. Lee JS. The effects of gender, obesity rate, nutrition knowledge and dietary attitude on the dietary self-efficacy of adolescents. Korean J Community Nutr. 2003;8(5):652-7.

15. Kahn HS, Tatham LM, Rodriguez C, Calle EE, Thun MJ, Heath CW Jr. Stable behaviors associated with adults' 10 -year change in body mass index and likelihood of gain at the waist. Am J Public Health. 1997;87(5):747-54

16. Parker DR, Gonzalez S, Derby CA, Gans KM, Lasater TM, Carleton RA. Dietary factors in relation to weight change among men and women from two southeastern New England communities. Int J Obes Relat Metab Disord. 1997:21(2):103-9.

17. Kohl HW $3^{\text {rd }}$, Hobbs KE. Development of physical activity behaviors among children and adolescents. Pediatrics. 1998;101(3 Pt 2):549-54.

18. Poirier P, Despres JP. Exercise in weight management of obesity. Cardiol Clin. 2003;19(3):451-70.

19. Wing RR. Physical activity in the treatment of the adulthood overweight and obesity: current evidence and research issues. Med Sci Sports Exerc. 1999;31(11 Suppl):S547-52.

20. Tremblay MS, Willms JD. Is the Canadian childhood obesity epidemic related to physical inactivity?. Int J Obes Relat Metab Disord. 2003;27(9):1100-5.

21. Swinburn B, Egger G. Preventive strategies against weight gain and obesity Obes Rev. 2002;3(4):289-301.

22. Shah P, Misra A, Gupta N, Hazra DK, Gupta R, Seth P, et al. Improvement in nutrition-related knowledge and behaviour of urban Asian Indian school children: findings from the 'Medical education for children/Adolescents for Realistic prevention of obesity and diabetes and for healthy aGeing' (MARG) intervention study. Br J Nutr. 2010;104(3):427-36

Article History: Submission Date : 01-06-2017; Revised Date : 04-09-2017; Acceptance Date : 11-09-2017.

Cite this article: Thamby SA, Sarriff AB, Ping NY, Nicholas D, Pandian G, Parachuri S. Effectiveness of an interventional educational tool on certain overweight and obesity parameters among the general public in Sungai Petani, Kedah DarulAman, Malaysia. J Young Pharm. 2017;9(4):588-92. 